\title{
UNA ESPECIE NUEVA DE TILLUS OLIVIER, 1790 (COLEOPTERA, CLERIDAE) DE LA PENÍNSULA IBÉRICA
}

\author{
P. Bahillo de la Puebla*, J. I. López-Colón** y M. García-París***(1)
}

\begin{abstract}
RESUMEN
Se describe Tillus ibericus sp. nov. de la Comunidad Autónoma de Madrid (España) y se compara con las otras especies del género Tillus Olivier, 1790 de la región Paleártica occidental. Tillus ibericus se distingue con facilidad de las especies próximas por la estructura del punteado elitral y por su coloración característica. La especie nueva comparte un aspecto general y caracteres de la estructura del cuerpo y de las antenas con $T$. flabellicornis Fairmaire, 1866 del Norte de África. Todas las citas anteriores de T. flabellicornis de la Península Ibérica corresponden a T. ibericus sp. nov.
\end{abstract}

Palabras clave: Taxonomía, Coleoptera, Cleridae, Península Ibérica, Tillus ibericus sp. nov.

\section{ABSTRACT \\ A new species of Tillus Olivier, 1790 (Coleoptera: Cleridae) from the Iberian Peninsula}

Tillus ibericus sp. nov. is described from the Comunidad Autónoma de Madrid (Spain) and it is compared to the western Palaearctic species of the genus Tillus Olivier, 1790. Tillus ibericus is easily distinguished from all other species of the genus by the structure of the elytral sculpture and its characteristic coloration. This new species shares a general habitus and characters from the antennae, and general body stucture with T. flabellicornis Fairmaire, 1866, a species from northern Africa. All preexisting records of T. flabellicornis in the Iberian Peninsula correspond to T. ibericus.

Key words: Taxonomy, Coleoptera, Cleridae, Iberian Peninsula, Tillus ibericus sp. nov.

\section{Introducción}

La familia Cleridae Latreille, 1802 se halla discretamente representada en la fauna paleártica con alrededor de 125 especies (Gerstmeier, 1998). En la Península Ibérica su conocimiento ha experimentado un resurgir en los últimos años, habiéndose publicado estudios faunísticos parciales referidos a determinadas áreas geográficas: Cataluña, Andalucía, Aragón, País Vasco y Navarra (Español, 1959; Bahillo de La Puebla \& López-Colón, 1999, 2001a; Bahillo de La Puebla et al., 1999), o a subfamilias o géneros concretos de esta familia (Español, 1960; Bahillo de La

* $\quad$ C/ Ibaizabal, $1,1^{\circ} \mathrm{C}$; E-48901 Barakaldo, Vizcaya, España.

** Plaza de Madrid, 2, $1^{\circ}$ D; E-28529 Rivas-Vaciamadrid, Madrid, España.

*** Museo Nacional de Ciencias Naturales, CSIC.; José Gutiérrez Abascal, 2; E-28006 Madrid, España. Autor para correspondencia 
Puebla \& López-Colón, 2001b). Además se ha recopilado un considerable número de registros dispersos repartidos en publicaciones entomológicas de carácter más general. Como resultado de todos estos estudios se hallan catalogadas en la actualidad un total de 34 especies de cléridos en la Península Ibérica.

De las 6 subfamilias presentes en la región Paleártica Occidental, la subfamilia Tillinae, caracterizada por presentar el pronoto sin reborde lateral, las cavidades coxales anteriores cerradas y los tarsos con el primer artejo claramente visible en observación dorsal y con el tercer y cuarto tarsómeros de similar desarrollo, incluye 4 géneros presentes en la Península Ibérica y áreas mediterráneas circundantes (Bahillo de La Puebla \& López-Colón, 2001b). Entre éstos se halla el género Tillus Olivier, 1790, que se caracteriza porque sus integrantes presentan la cabeza con un desarrollo normal, sin un alargamiento marcado; tienen el último antenómero, a lo sumo, tan largo como los dos precedentes juntos; los élitros, que en general cubren completamente el abdomen, muestran su anchura máxima en el tercio posterior y son relativamente largos, más de dos veces más largos que anchos, cuando se consideran en conjunto y la anchura se mide a la altura de la región humeral.

Tillus está representado por cinco especies en la región Paleártica Occidental: Tillus elongatus (Linnaeus, 1758), T. pallidipennis Bielz, 1850, T. mozabitus Chobaut, 1897, T. pectinicornis Abeille, 1892 y T. flabellicornis Fairmaire, 1866 (Corporaal, 1950; Gerstmeier, 1998). Todas las especies son en general poco frecuentes. Únicamente T. elongatus coloniza una amplia región de la Península Ibérica, aunque incluso a pesar de ser la especie más extendida, solamente presenta registros dispersos en el tercio septentrional (Bahillo de La Puebla \& LópezColón, 2001b).

García-París et al. (2001) citaron por primera vez T. flabellicornis en la Península Ibérica a partir de un ejemplar capturado en Madrid. Posteriormente, Bahillo de La Puebla \& LópezColón (2001b), en una revisión de los Tillinae de la Península Ibérica estudiaron otros tres ejemplares del mismo taxon procedentes también de Madrid. El examen detallado de estos ejemplares y su comparación con ejemplares de las especies próximas del género demuestran que las características morfológicas que separan a estos ejemplares del resto de especies conocidas son constantes y que por lo tanto nos encontramos ante una especie nueva que describimos a continuación.
Tillus ibericus sp. nov. (Fig. 1a)

Holotipo: España: Madrid: Villanueva de Perales, 5-VI-2000, $1 \sigma^{\top}$ (M. García París leg.), depositado en el Museo Nacional de Ciencias Naturales, CSIC, Madrid.

PARATIPos: España: Madrid: Tres Cantos, 27-V-1996, $10^{7}$ y 1

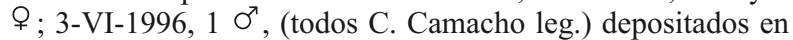
las colecciones C. Camacho y Bahillo-López Colón

Diagnosis: Tillus con élitros tricolores, con la mitad basal de color castaño rojizo uniforme y la mitad apical de color negro con una banda transversal blanquecina que, partiendo del borde externo de cada élitro, no alcanza la sutura elitral; con punteado elitral grueso, cuyo diámetro se mantiene más o menos constante desde la región humeral hasta el borde posterior de la banda blanca transversal, a partir del cual disminuye drásticamente.

DESCRIPCIÓN: Longitud total de 6 a $10 \mathrm{~mm}$ (Figura 1a). Ligero dimorfismo sexual que afecta a la conformación de las antenas, que son aserradas en las hembras y pectinadas en los machos.

Cabeza más ancha que el borde anterior del pronoto, convexa y con un punteado muy fino sobre el cual aparece una pubescencia larga, negra y erecta. Ojos moderadamente prominentes, reniformes, pubescentes y escotados en la proximidad de las inserciones antenales. Antenas de once artejos que no alcanzan la base de los élitros.

Pronoto completamente negro, más ancho en el borde anterior que en el posterior, con dos protuberancias muy marcadas en los lados; región central deprimida transversalmente; borde posterior manifiestamente rebordeado con una pequeña depresión triangular en la zona central. Los tercios basal y apical del pronoto presentan un punteado de pequeño diámetro y muy denso, con el espacio que separa puntos contiguos de menor tamaño que el diámetro de los puntos; en el tercio central y especialmente en la parte anterior de la depresión transversal, el punteado se transforma en arrugas transversas (Figura 1a). Sobre el punteado pronotal aparece una pubescencia muy larga, negra y erecta.

Escudete negro, densamente punteado y pubescente.

Élitros dos veces y media más largos que anchos, considerados en conjunto y con la anchura medida a la altura de la región humeral; claramente ensanchados en la región preapical y tricolores, con la mitad anterior castaño-rojiza y la mitad posterior negra, presentando entre ambas una banda blanquecina transversal, curvada; dicha banda progresa desde el borde exterior de cada élitro hacia la sutura, pero no llega a alcanzarla. El punteado elitral está muy marcado; desde el borde basal hasta 

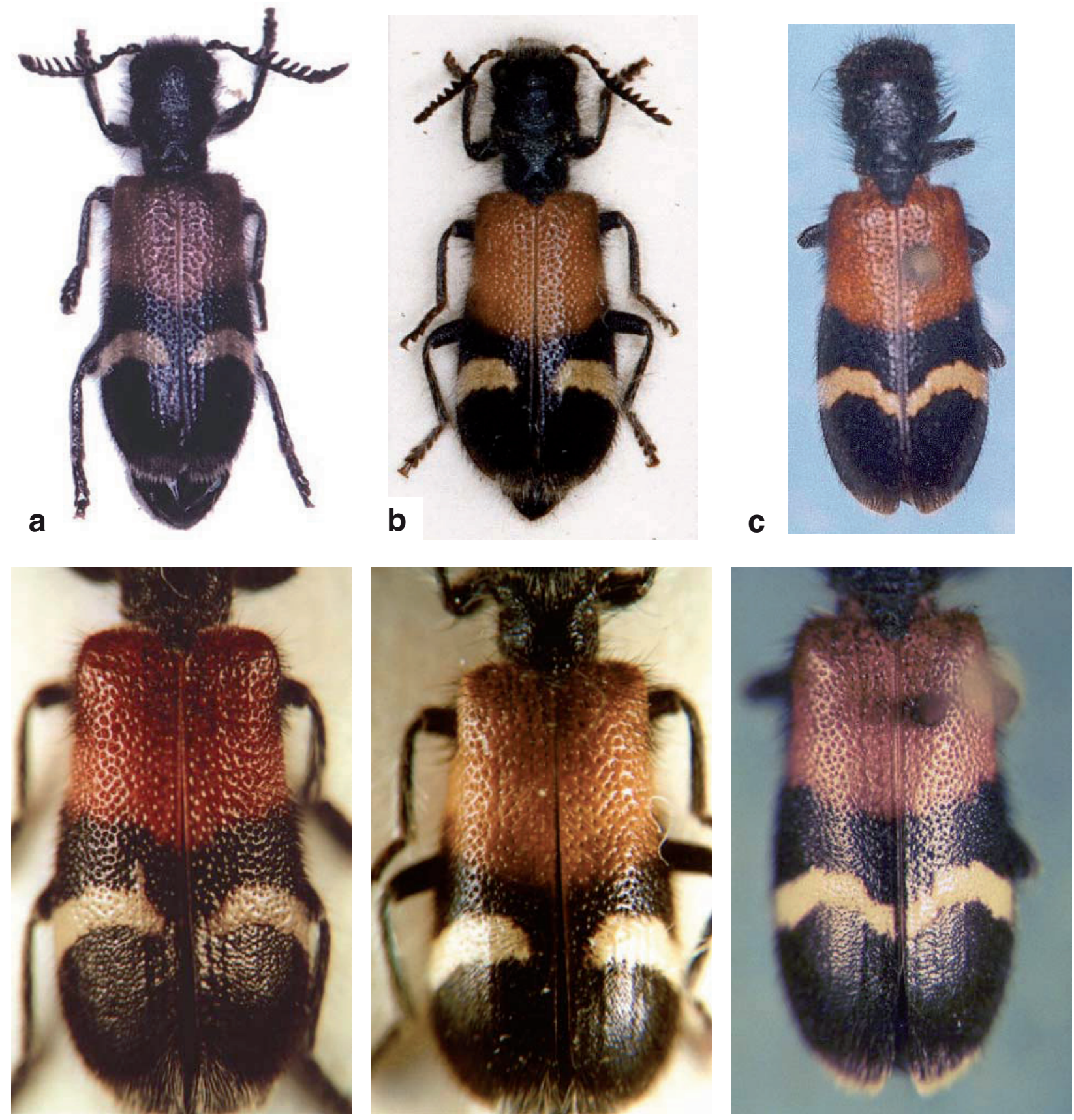

Fig. 1.- Habitus, arriba, y detalle del punteado elitral, abajo, de (a) Tillus ibericus sp. nov. Holotipo (España: Madrid: Villanueva de Perales); (b) Tillus flabellicornis Fairmaire, 1866 (Marruecos: Ketama-Rift: Bab Chiquer); (c) Tillus pectinicornis Abeille, 1892 (Akhbes).

Fig. 1.- Habitus, above, and close up of the elitral sculpture, below, of (a) Tillus ibericus sp. nov. Holotipus (España: Madrid: Villanueva de Perales); (b) Tillus flabellicornis Fairmaire, 1866 (Marruecos: Ketama-Rift: Bab Chiquer); (c) Tillus pectinicornis Abeille, 1892 (Akhbes). 

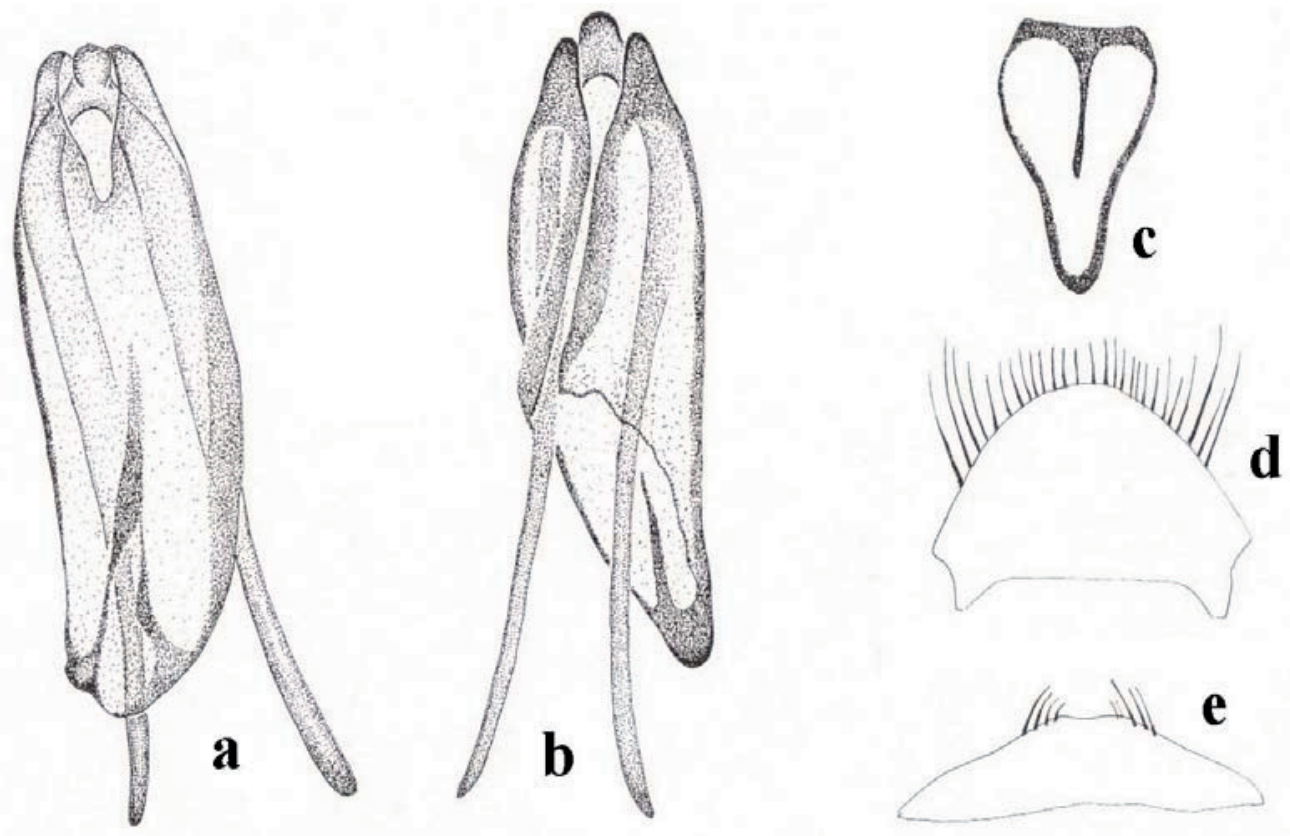

Fig. 2.- Tillus ibericus sp. nov. (a) Edeago, vista dorsal; (b) idem, vista ventral; (c) spiculum gastral; (d) pigidio; (e) ápice del VII esternito abdominal.

Fig. 2.- Tillus ibericus sp. nov. (a) Aedeagus, dorsal view; (b) idem, ventral view; (c) spiculum gastrale; (d) pigidium; (e) apex of the VII abdominal esternite.

la banda blanquecina el punteado es irregular y denso, con los puntos de gran tamaño y con un diámetro que se mantiene prácticamente constante desde el borde basal hasta el borde posterior de la banda blanquecina transversal; a partir del borde posterior de dicha banda el diámetro de los puntos cambia drásticamente haciéndose más pequeño; en esta zona posterior el punteado mantiene su diámetro pero se hace más confuso, tomando una apariencia rugosa (Figura 1a). Del punteado elitral parte una pubescencia densa, inclinada hacia atrás y negra, excepto en la banda blanquecina transversal, en la que las sedas son también blancas. En el área basal de los élitros, especialmente en la región periescutelar, la pubescencia es notablemente más densa; a lo largo de la sutura se observan sedas erectas más largas que sobresalen del resto de la pubescencia.

Patas negras, relativamente cortas, con pubescencia doble blanco-amarillenta: una corta, inclinada hacia atrás, y otra más larga y escasa, perpendicular. Los tarsos son pentámeros y el último artejo presenta el ápice más claro. Las uñas están bifurcadas en el extremo.
El pigidio es regularmente redondeado y presenta en el VII esternito abdominal una ligera escotadura apical (Fig. 2d y e).

La genitalia masculina se representa en la figura 2. Lo más característico de ella es la particular torsión del tegmen con respecto al eje longitudinal del pene.

ETIMOLOGÍA: El nombre de la especie es un adjetivo latino que hace referencia a la Península Ibérica, en cuya zona centro se sitúan los enclaves de procedencia de los ejemplares que componen la serie típica.

Biología: Desconocida. El ejemplar de Villanueva de Perales fue capturado en el mes de junio, en un día soleado, sobre Retama sphaerocar$p a$, en un retamar con encinas dispersas (GarcíaParís et al., 2001); los otros tres ejemplares eclosionaron de ramas muy secas de encina (Quercus ilex), donde presumiblemente depredarían sobre insectos xilófagos, como hacen otros componentes del género (Bahillo de La Puebla \& López-Colón, 2001b).

DistribuCión: Por el momento sólo se tiene constancia de su presencia en la Comunidad 
Autónoma de Madrid (centro de la Península Ibérica).

Comentarios taxonómicos: Como se expuso anteriormente, los ejemplares examinados, y que componen la serie típica fueron asignados previamente a la especie Tillus flabellicornis (GarcíaParís et al., 2001; Bahillo de La Puebla \& LópezColón, 2001b); por lo tanto, esta especie debe borrarse del catálogo ibérico.

\section{Discusión}

El nuevo taxon se diferencia fácilmente de visu del resto de las especies del género Tillus presentes en la región Paleártica occidental. De Tillus elongatus y de $T$. pallidipennis, especies ampliamente distribuidas en el centro y este de Europa, se diferencia porque estas especies presentan los élitros monocolores, negro-azulados la primera y amari1lo-ocráceos la segunda, mientras que en T. ibericus son tricolores. De T. mozabitus, taxon tan sólo conocido de Argelia, se diferencia porque la cabeza y patas de esta especie son de color castaño, frente a la coloración negra que exhiben la cabeza y patas de T. ibericus. De T. pectinicornis, especie propia de Creta y Oriente Medio, se diferencia por el patrón de punteado elitral. En T. pectinicornis el diámetro de los puntos se reduce progresivamente desde la base hacia el ápice, mientras que en Tillus ibericus el diámetro de los puntos se mantiene constante desde la base hasta el borde posterior de la banda blanca a partir de donde se reduce llamativamente. Además, en los ejemplares de T. pectinicornis que hemos podido observar, la banda blanquecina transversal alcanza la sutura elitral, mientras que en T. ibericus no lo hace (Figura 1c). De $T$. flabellicornis, especie conocida de Argelia y Marruecos, se diferencia porque en éste el punteado elitral es menos grueso y no alcanza el borde posterior de la banda blanquecina transversal. En el nuevo taxon, el punteado elitral es apreciablemente más grueso y se extiende por toda la banda transversal blanquecina. Además, la mitad anterior de los élitros de T. ibericus es de un color castaño-rojizo uniforme, mientras que en T. flabellicornis esta zona es netamente más clara, de color amarillopajizo (Figura 1b).

Tanto por la forma y tamaño de las antenas, por la estructura general del cuerpo y por el patrón de coloración, T. ibericus, resulta mucho más afín a la especie norteafricana $T$. flabellicornis que al resto de especies.
La Comunidad Autónoma de Madrid es sin duda una de las regiones de la Península Ibérica mejor muestreada desde el punto de vista entomológico y sin embargo siguen apareciendo especies nuevas de coleópteros localizadas a pocos kilómetros de la ciudad de Madrid (López-Colón, 1997; Ortuño, 1997, 2000). Algunas de estas especies pueden corresponder al resultado de nuevas evaluaciones taxonómicas o al descubrimiento de especies de pequeño tamaño poco llamativas. En otros casos se trata de especies de colores vistosos y relativo gran tamaño, pertenecientes a grupos bien conocidos que simplemente han escapado a la atención de naturalistas y entomólogos. En el caso que nos ocupa, los primeros ejemplares de T. ibericus se encontraron en 1996 y posteriormente en 2000, sin que se haya localizado ningún ejemplar colectado con anterioridad en las colecciones históricas de los museos. Su aparente similitud con los otros taxones de parecida coloración de la cuenca mediterránea como T. pectinicornis y T. flabellicornis y la ausencia de suficiente material de comparación llevó de forma conservadora a su inclusión inicial bajo la denominación de T. flabellicornis (García-París et al., 2001).

En cualquier caso resulta sorprendente el hallazgo de este nuevo taxon en Madrid ya que todas las especies euromediterráneas del género se describieron antes de 1900. Ignoramos las razones por las que esta llamativa especie ha pasado desapercibida, aunque podríamos suponer que o bien se trata de una especie sumamente rara cuya densidad poblacional se haya disparado durante la década anterior o bien que la especie haya colonizado recientemente la región desde otras áreas del centro peninsular menos prospectadas. Es posible que un cambio climático haya favorecido una mayor actividad y dispersión de la especie, ya que, precisamente las especies más afines a T. ibericus ocupan zonas relativamente cálidas del Mediterráneo oriental y del norte de Africa (T. flabellicornis y T. pectinicornis).

La existencia de cambios a gran escala en las distribuciones geográficas motivados por alteraciones climáticas, podría determinarse mediante la comparación de los resultados de campañas de muestreo antiguas, con los resultados de campañas actuales. En el caso de Madrid la existencia de colecciones de diversa índole, incluidas las colecciones históricas, podría facilitar este tipo de comparaciones, especialmente para aquellos grupos en los que se ha trabajado de forma más o menos continuada a lo largo del tiempo, como quizás sea el caso de algunos Lepidoptera (ropalóceros) (Gómez de Aizpurua, 1987) y Coleoptera (Carabidae) (García-París et al., 
2001; Ortuño \& Toribio, 1996). Desafortunadamente en el caso de la familia Cleridae estos datos no existen por lo que la mayoría de las observaciones, incluidas las que aquí nos ocupan, han de considerarse simplemente anecdóticas.

\section{AGRADECIMIENTOS}

Dejamos constancia de nuestro agradecimiento a $\mathrm{C}$ Camacho (Tres Cantos, Madrid) y J. L. Zapata (Tres Cantos, Madrid) que nos facilitaron el estudio de los paratipos que se mencionan. A M. A. Zarazaga y V. M. Ortuño sus oportunos comentarios al manuscrito. A M. París y C. Martín las facilidades prestadas para el estudio de los ejemplares de T. flabellicornis y T. pectinicornis conservados en el Museo Nacional de Ciencias Naturales (Madrid). Agradecemos a la Comunidad de Madrid los permisos correspondientes para la realización de los muestreos en los aledaños de la carretera M-501 donde fue encontrado T. ibericus.

\section{Referencias}

Bahillo de la Puebla, P. \& López-Colón, J. I., 1999. Cléridos de Aragón (Coleoptera, Cleridae). Insecta: Coleoptera. Familia 37. Catalogus de la entomofauna aragonesa, 20: 3-11.

BAhillo de la Puebla, P. \& LóPez-Colón, J. I., 2000. El género Opilo Latreille, 1802 en la Península Ibérica (Coleoptera, Cleridae). Boletín de la Asociación española de Entomología, 24(1-2): 213-227.

BAhillo de la Puebla, P. \& LóPez-Colón, J. I., 2001a. Cléridos de Andalucía (Coleoptera, Cleridae). Manuel Baena Ruiz, Delegación de Cultura del Excmo. Ayuntamiento de Utrera, Fundación El Monte, Sociedad Andaluza de Entomología. Utrera. 77 pp.

BAhillo de la Puebla, P. \& LóPez-Colón, J. I., 2001b. La subfamilia Tillinae Leach, 1815 (Coleoptera, Cleridae) en la Península Ibérica. Estudios del Museo de Ciencias Naturales de Álava, 16: 153-171.

Bahillo de la Puebla, P., Recalde Irurzun, I., San Martín Moreno, A. F. \& López-Colón, J. I., 1999. Contribución al conocimiento de los cléridos de la Comunidad Autónoma Vasca, Comunidad Foral Navarra y áreas limítrofes. (Coleoptera, Cleridae). Estudios del Museo de Ciencias Naturales de Álava, 14: 151-167.

Corporate, J. B., 1950. Cleridae. Coleopterorum Catalogus, Supplementa pars 23, Uitgeverij Dr. W. Junk's. Gravenhage. 373 pp.

EsPAÑol, F., 1959. Los Cléridos (Cleridae) de Cataluña e Islas Baleares (Col., Cleroidea). Publicaciones del Instituto de Biología aplicada (Barcelona), 30: 105-146.
EsPAÑOL, F., 1960. Los Trichodes ibéricos (Col. Cleridae). Graellsia, 18: 153-164.

García-París, M., BAhillo de la Puebla, P. \& LóPezColón, J. I., 2001. Tillus flabellicornis Fairmaire, 1866 (Coleoptera, Cleridae), primera cita para Europa. Biocosme Mésogéen, 18(1): 23-26.

García-París, M. \& París, M., 1993. Distribución de los Carabinae (s.str.) (Col. Carabidae) de Madrid: atlas provisional. Boletín de la Asociación Española de Entomología, 17(2): 27-36.

Gerstmeier, R., 1998. Checkered Beetles. Illustrated key to the Cleridae of the Western Palaearctic. Margraf Verlag. Wekersheim. 241 pp. + 8 Láms.

Gómez dE AizpuruA, C., 1987. Atlas provisional. Lepidópteros de Madrid. II. Consejería de Agricultura y Ganadería de la Comunidad de Madrid. Madrid. 101 pp.

López-Colón, J. I., 1997. Plagionotus marcae n. sp., nueva especie del centro de la Península Ibérica (Coleoptera: Cerambycidae). Lambillionea, 97(2): 219-233.

Ortuño, V. M. \& Toribio, M., 1996. Los Coleópteros Carábidos. Morfología, biología y sistemática. Fauna de la Comunidad de Madrid. Organismo Autónomo Parques Nacionales. Ministerio de Medio Ambiente. Madrid. 269 pp.

ORTUÑO, V. M., 1997. Description of a new Hypotyphlus Jeannel from the Sistema Central Mountain Range in Spai and comments on Hypotyphlus guadarramus (Ehlers) (Coleoptera: Trechidae). The Coleopterist Bulletin, 51: 217-221.

OrtuÑo, V. M., 2000. Nueva especie de Typhlocharis Dieck, 1869, de la Península Ibérica (Coleoptera, Carabidae, Trechinae). Bulletin de la Société entomologique de France, 105: 279-284. 